\title{
Stochastic Model Updating of Bolt-Jointed Structure for Structural Dynamics Applications
}

\author{
M.A.S. Aziz Shah', M.A. Yunus1,,, M.N. Abdul Rani', R. Omar² and Akhil Mora ${ }^{3}$ \\ ${ }^{1}$ Structural Dynamics Analysis and Validation (SDAV),Faculty of Mechanical Engineering, Universiti Teknologi MARA (UiTM), 40450 Shah Alam, \\ Selangor, Malaysia \\ ${ }^{2}$ Machinery Technology Center, SIRIM Industrial Research, 44200, Hulu Selangor, Selangor, Malaysia \\ 33MSC Software, \#5AC, 961, Service Rd, 1st Block, Bank Avenue Colony, Horamavu, Bengaluru, Karnataka 560043, India
}

\begin{abstract}
Structural stiffness exerts from joint connections and contact interfaces are significantly affect the dynamic behaviour of the bolt-jointed structure. Randomness in the joint connections due to the manufacturing variability in the identical bolted joints and uncertainty in contact interfaces due to the assambled and reassambled of the joint structure make sets of the dynamic behaviour of the bolt-jointed structure always inconsistent. On this account, a stochastic analytical model needs to be developed for the bolt-jointed structure to be used for uncertain parameters quantification. Hence, this paper is intended to propose an accurate and efficient stochastic analytical modelling of bolt-jointed structure in predicting the dynamic behaviour of the structure due to the randomness in the joint connections and uncertainty in contact interfaces. The aim of the study was accomplished by investigating four different finite element (FE) models of boltjointed structure with different element connectors to represent the bolted joints connections, namely rigid element (RBE), beam element (CBEAM), and 2 types of spring elements namely CELAS and CBUSH. Stochastic modelling was conducted by coupled the appropriate FE models with Latin Hypercube Sampling (LHS) algorithm to provide variability sampling due to the randomness in the bolted joints. The experimental modal analysis was performed by reassembled and disassembled the bolted joints to extract the variability in the dynamic behaviour, and the results were compared with LHS using statistical characteristics. Stochastic model updating then was used to minimise the discrepancies between experimental result and predicted model. The result has shown that the $\mathrm{CBUSH}$ is the most appropriate connector to accurately predict the dynamic behaviour of the bolt-jointed structure under variability conditions using the stochastic model updating method.
\end{abstract}

ARTICLE HISTORY

Received: $10^{\text {th }}$ Dec 2020

Revised: $11^{\text {th }}$ May 2021

Accepted: $14^{\text {th }}$ June 2021

\section{KEYWORDS}

Bolted-joint;

Contact interface;

Stochastic model

updating;

Uncertainty quantification

\section{INTRODUCTION}

Bolt joints are widely used in the mechanical industries, such as in the applications of automotive engine, pipe flanges, and machining pulley joint. The vibration effects on the bolt-jointed structure have become an important criterion in deciding the level of safety and economical for industrial productions. However, accurate analytical modelling of boltjointed structure has always been found to be very difficult and cumbersome [1], [2]. The accurate responses obtained from analytical modelling is highly dependent on the structural model form and structural model parameters [3]. These criteria make the analytical modelling, especially using the finite element (FE) method becomes a challenging task. In the bolt-jointed structure, the modelling of the substructures, joint interfaces and contact zone are the three main characteristics that affect the reliability of the predicted responses.

In recent, a large amount of research has been reported for the modelling of bolted joint [4], and they suggested the advantages of the solid bolt model in which accurate predicted responses of the structure could be obtained. However, the solid bolt model causing an extra $38 \%$ of excessive computational time and seems to be unpractical to be used for a high amount of substructure connections [5]. Besides, there are several cases that are used 1D element connectors in which much more efficient to be used to represent bolts [6].

Generally, analytical modelling of bolt-jointed structure using finite element method (FEM) for vibration analysis is usually constructed and optimised by considering deterministic parameters, in which experimental data from a single structure is employed to update a single FE model by minimising the residual between predicted results and experimental data [7]. The deterministic procedure and solution are only allowed to be conducted if the problem is assumed to be no variability exited. Various works have been put forward to optimise the initial FE model of bolted joint modelling using the deterministic model updating method. For instance, Ahdian et al.[8] implement model updating method for assembled structure consists of bolted lap joint using a sensitivity method. Zhi et al. [9] developed a parametric model of bolt aero engine and optimised the model with multi behaviour responses. Omar et al. [10] implement the model updating method to improve initial model of bolt joints with consist of stiffness members, bolt representation and joint's affected areas. 
However, in actual application, bolt-jointed structures are usually inherent in uncertain parameters. Besides, different sources of uncertainty usually arise in the system and contributed to the high variability in the experimental responses, which is the dynamic behaviour of the structures. The major sources of uncertainty that contribute to the variability in the bolt-jointed structure responses are when the bolt joints disassembled and reassembled repeatedly, self-loosening due to vibration and slippage [11], [12].

In order to consider the variability effects that exist due to the randomness or uncertainties in structural parameters and joint connections, stochastic model updating (SMU) has been explored recently. The SMU method is developed based on statistical properties. The predicted statistical properties parameters are optimised and altered to match their experimental counterparts by using an uncertainty inverse propagation scheme [13]. Moreover, the variability in experimental data can be examined in SMU as suggested by Patelli et al. [14], in which several sets of experimental configurations for the dynamic behaviour of assembled bolt-jointed structure are taken into account. To support the works, a study by Husain et al. [15] highlights the stochastic method allows for robust and credible FE models to be produced, which in turn increase confidence in the design and analysis of structures.

This paper focuses on developing an accurate stochastic dynamic behaviour of bolt-jointed structure with concerning to model source of uncertainty contributed from stiffness properties of bolt joints interface and contact zones. Research works from this paper were describe into three stages:

i. Stage 1: Finite element analysis, experimental modal analysis and model updating for individual substructures,

ii. Stage 2: Finite element analysis, experimental modal analysis for assembled bolt-jointed structure, and finally

iii. Stage 3: Stochastic finite element modelling and validation (updating) for bolt-jointed structure.

In this study, Stage 1 was conducted to minimise structural uncertainties that may contribute to high variability in the dynamic behaviour of the bolt-jointed structure. Then, in Stage 2, the identification of the most appropriate models between FE-Type 1, FE-Type 2, FE-Type 3 and FE-Type 4 (discussed further in subsection STAGE 2) to represent the bolt-jointed structure was conducted and finally, Stage 3 was conducted to predict, analyse and update the bolt-jointed structure using stochastic model updating method and propagated the uncertainty of parameters.

\section{RESEARCH METHODOLOGY}

\section{Stage 1: Development of Accurate FE Model For Individual Substructures}

\section{Finite element modelling of individual substructures}

In this work, an assembly of the bolt-jointed structure is used. The structure consists of two identical plates with an elliptical hole shape called elliptical plate A and elliptical plate B; were assembled using four M10 bolts and nuts. The plates were fabricated with the same geometry proportion with overall dimensions are; length of $380 \mathrm{~mm}$, a width of 45 $\mathrm{mm}$ and a thickness of $6 \mathrm{~mm}$. The structure was designed to represent complex dynamic responses that commonly experienced from machine structures in various mechanical industries. The material used for elliptical plate A and elliptical plate B is mild steel, and the material data is tabulated in Table 1. The FE models of the substructures are shown in Figure 1 were modelled using 3D Tetra elements in MSC Patran software with consists of 30016 nodes and 23754 elements for elliptical plate A; meanwhile, 29484 nodes and 23286 elements for elliptical plate B. Solution 103 in MSC Nastran was used to calculate the dynamic behaviour of the substructures.

Table 1. Material properties of substructures [16].

\begin{tabular}{lcc}
\hline Parameters & Elliptical plate A & Elliptical plate B \\
\hline Young's modulus, E & $200 \mathrm{GPa}$ & $200 \mathrm{GPa}$ \\
Poisson's ratio, $v$ & 0.30 & 0.30 \\
Mass, $m$ & $0.735 \mathrm{~kg}$ & $0.732 \mathrm{~kg}$ \\
Density, $\rho$ & $7937.35 \mathrm{~kg} / \mathrm{m}^{3}$ & $7904.95 \mathrm{~kg} / \mathrm{m}^{3}$ \\
\hline
\end{tabular}

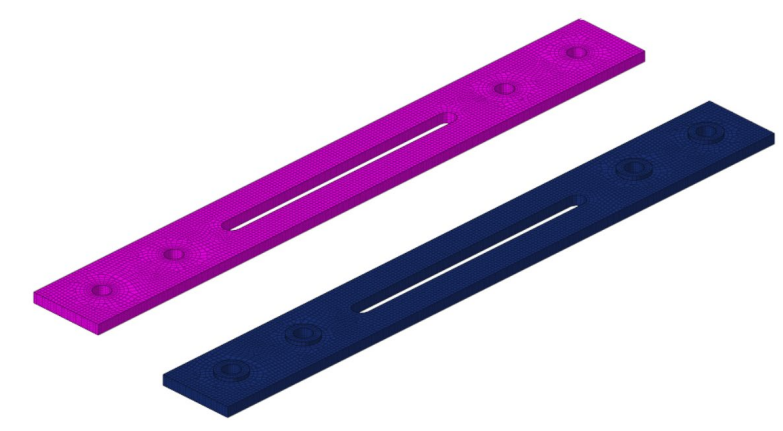

Figure 1. Substructures elliptical plate A and elliptical plate B. 
The NASTRAN Solution 103 is based on normal modes analysis. The equation is established by using the equation of motion expressions:

$$
\mathbf{M} \ddot{x}+\mathbf{C} \dot{x}+\mathbf{K} x=0
$$

where $\mathbf{M}$ and $\mathbf{K}$ are $n \times n$ mass, and stiffness matrices and the system are calculated based on the structure properties of the models. In the normal modes analysis, the matrix damping $(\mathbf{C})$ has always been ignored since the structures are considered as undamped structure [17]. Hence the equation of motion is reduced to:

$$
\mathbf{M} \ddot{x}+\mathbf{K} x=0
$$

The equation of motion for undamped free vibration of the substructures can be expressed as:

$$
\left[\mathbf{K}-\boldsymbol{\lambda}_{\mathbf{j}} \mathbf{M}\right] \boldsymbol{\phi}_{\mathbf{j}}=0
$$

where $\boldsymbol{\phi}_{\mathbf{j}}$ is the $\mathrm{n} \times 1$ modal displacement vector (mode shapes), $\boldsymbol{\lambda}_{\mathbf{j}}=\boldsymbol{\omega}^{2}$ is the eigenvalue, and $\boldsymbol{\omega}$ is the natural frequencies of the substructures.

\section{Experimental modal analysis of individual substructures}

Experiment modal analysis (EMA) is a method that actively used by researchers to measure structural response such as dynamic behaviour of a mechanical structure. The dynamic behaviour can be classified as the natural frequencies and mode shapes of a structure. In this work, EMA was conducted on each substructure (elliptical plate A and elliptical plate B) with free-free boundary conditions. The impact hammer and roving accelerometers methods were used in this test. Prior to the test, the substructures were discretised into 18 points, and from the 18 points, one 1 point was used as excitation point; meanwhile, another 17 points were used as measuring points with first ten modes. The locations of excitation and measuring points were chosen since there are not located at any nodal points [18]. Moreover, since the mass of each accelerometer was $1.5 \mathrm{~g}$ and the elliptical plate A and elliptical plate B were $734 \mathrm{~g}$ and $732 \mathrm{~g}$, respectively, there are no mass loading issues in the use of accelerometers for both tests. This is because the mass effects in these tests were below 1/10.

The substructures were measured using 16 channel LMS SCADAS acquisition system. The dynamic behaviour such as natural frequencies and mode shapes of the substructures were extracted using PolyMAX curve fitting method from the LMS software. Figure 2 shows the experimental setup of the substructures.

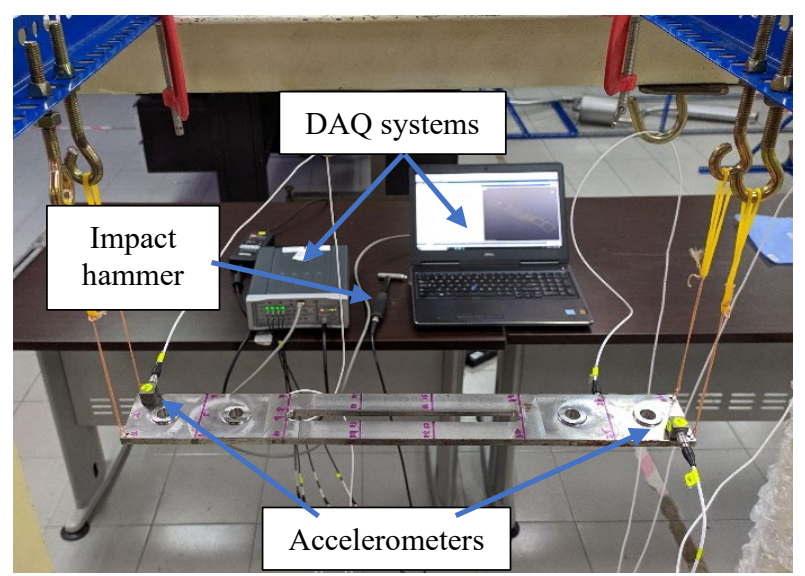

Figure 2. Experimental setup for substructure.

\section{Model updating of individual substructures}

Model updating is used to refine the accuracy of the FE models against EMA data by minimising the discrepancies between the results in which the influence parameters are adjusted systematically within the permitted range [19]. The model updating method was employed in order to minimise the discrepancies in the dynamic behaviour of the substructures as a result of uncertainties in the FE modelling, particularly in the input model properties. The updated parameters were selected systematically using sensitivity analysis.

It is essential to update the substructures to give an accurate modelling of substructures prior proceed with the FE modelling of the complete bolt-jointed structure. This effort is mainly to reduce the modelling uncertainties on the assembled structure. Therefore, in the next stage, modelling error and optimisation can be focusing on the bolt-jointed modelling. In this work, Solution 200 (Optimisation code) of MSC NASTRAN was used to perform updating process for each substructure of elliptical plate A and elliptical plate B. The first ten natural frequencies were integrated into the objective function as follow: 


$$
\mathbf{Z}_{\mathrm{m}}-\mathbf{Z}_{\mathbf{j}}=\mathbf{S}_{\mathbf{j}}\left(\boldsymbol{\theta}_{\mathbf{j}+\mathbf{1}}-\boldsymbol{\theta}_{\mathrm{j}}\right)
$$

where $\mathbf{Z}_{\mathbf{m}}$ and $\mathbf{Z}_{\mathbf{j}}$ is the vector of measured and predicted output, respectively, $\mathbf{S}_{\mathbf{j}}$ is the sensitivity matrix, and $\boldsymbol{\theta}_{\mathbf{j}}$ is the vector of system parameters. The sensitivity matrix is the first derivative of the eigenvalue and eigenvector with respect to the system parameters. By calculated the derivative of the $j$-th eigenvalue, $\boldsymbol{\lambda}_{\mathbf{j}}$, with respect to the $\mathbf{j}$-th parameter, $\boldsymbol{\theta}_{\mathbf{j}}$, the eigensystems can be written as

$$
\frac{\partial \lambda_{j}}{\partial \theta_{j}}=\boldsymbol{\phi}_{i}^{T}\left(\frac{\partial \mathbf{K}}{\partial \theta_{j}}-\lambda_{i} \frac{\partial \mathbf{M}}{\partial \theta_{j}}\right) \boldsymbol{\phi}_{i}
$$

The eigensystem, further, can be simplified in the form of natural frequencies using minimisation based least square method. The equation can be rewrite as follow

$$
\min \sum_{i=1}^{m} W_{i}\left(\frac{\omega_{i}^{n}}{\omega_{i}^{e}}-1\right)^{2}
$$

where $\omega_{i}^{n}$ is the FE frequencies, $\omega_{i}^{e}$ is the test frequencies, meanwhile $W_{i}$ is the equation weightage.

\section{Stage 2: Development of Accurate FE Model For Assembled Bolt-Jointed Structure}

\section{Finite element modelling of assembled bolt-jointed structure}

The FE models of the assembled bolt-jointed structure were developed as shown in Figure 3 and Figure 4. The FE models of the assembled structure were developed as extended from the updated FE models of substructure elliptical plate A and elliptical plate B. It is to minimise the structure uncertainties; hence, any discrepancies from the assembled FE models can be blamed on the bolted joint interfaces. There are four types of FE models of the assembled structure with different element connector to represent as bolt shank interfaces such as rigid element (RBE), beam element (CBEAM) and two spring elements (which are CELAS and CBUSH). The FE models were developed using MSC PATRAN software. Properties of the bolt joints (carbon steel) are tabulated in Table 2, in which the values were obtained using swift formulation [20].

Meanwhile, properties of the contact zone are tabulated in Table 3. Each of the models is identified as RBE boltjointed FE model (FE Type 1), CBAR bolt-jointed FE model (FE Type 2), CELAS bolt-jointed FE model (FE Type 3) and CBUSH bolt-jointed FE model (FE Type 4), respectively. The selections of the element connectors were based on the literature studies in which these connectors have been widely used by engineers and researchers to model bolt-jointed structure for various applications. In this study, Solution 103 in MSC NASTRAN were used to calculate the dynamic behaviour of the assembled FE models. The feasibility to represent as an analytical bolt-jointed structure between four FE models were identified by comparing the predicted responses obtained with experimental data of assembled structure. Then, only the most appropriate FE model is selected for the model updating process.

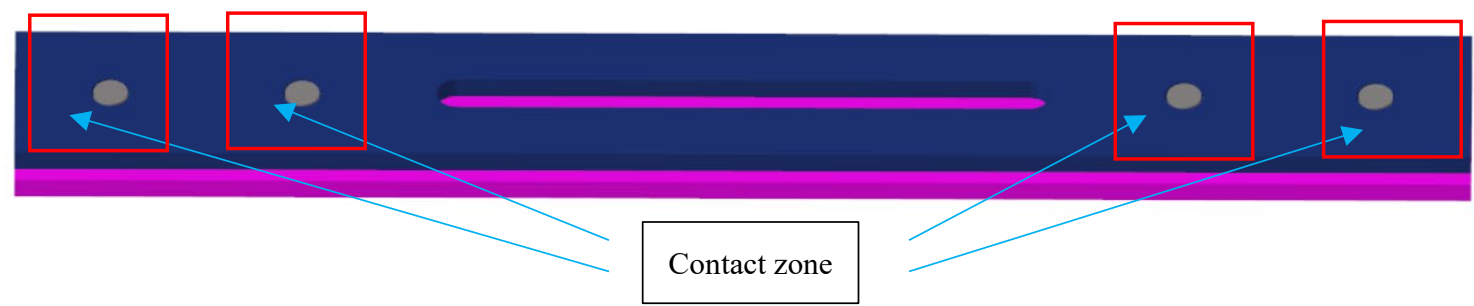

Figure 3. CAD model of the bolt-jointed structure.

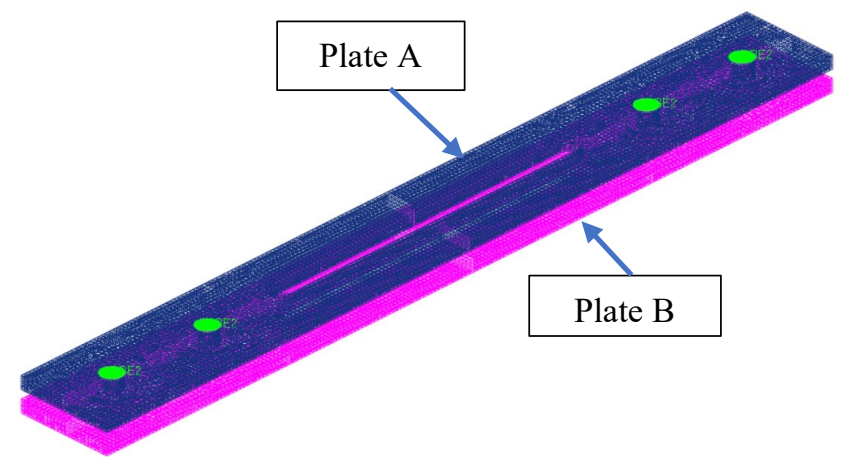

(a)

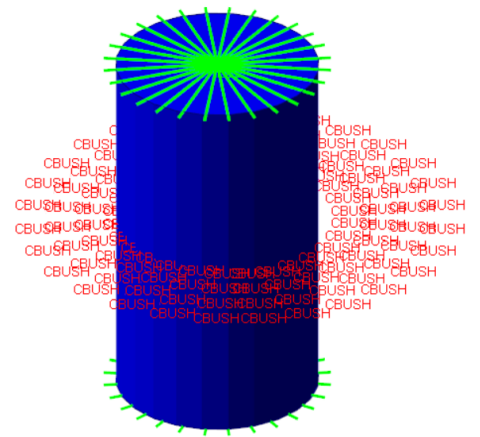

(b)

Figure 4. (a) FE model of the assembled structure and (b) bolt joint. 
Table 2. Material properties of bolt joints [20].

\begin{tabular}{lcccc}
\hline Parameters & Bolt 1 & Bolt 2 & Bolt 3 & Bolt 4 \\
\hline Young's modulus, $\mathrm{E}_{\mathrm{B}}(\mathrm{GPa})$ & 193 & 193 & 193 & 193 \\
Shear modulos, $\mathrm{G}_{\mathrm{B}}(\mathrm{GPa})$ & 78 & 78 & 78 & 78 \\
Poisson ratio, $\mathrm{Nu}_{\mathrm{B}}$ & 0.27 & 0.27 & 0.27 & 0.27 \\
Density, $P_{\mathrm{B}}\left(\mathrm{kg} / \mathrm{m}^{3}\right)$ & 8030 & 8030 & 8030 & 8030 \\
\hline
\end{tabular}

Table 3. Stiffness properties for bolted contact zone.

\begin{tabular}{lcccc}
\hline Parameters & Bolt 1 & Bolt 2 & Bolt 3 & Bolt 4 \\
\hline Axial - Z stiffness, $\mathrm{K}_{1}(\mathrm{MPa})$ & 56 & 56 & 56 & 56 \\
Shear - X stiffness, $\mathrm{K}_{2}(\mathrm{MPa})$ & 47 & 47 & 47 & 47 \\
Shear - Y stiffness, $\mathrm{K}_{3}(\mathrm{MPa})$ & 47 & 47 & 47 & 47 \\
\hline
\end{tabular}

\section{Experimental modal analysis of assembled bolt-jointed structure}

The bolt-jointed structure shown in Figure 5 was tested using impact hammer, and roving accelerometers methods with one excitation point and 35 measuring points covered all frequency of interest. The frequencies of interest for the assembled test structure was sets for the first ten natural frequencies. In this work, the structure was measured in two directions (of x-direction and z-direction). Multiple directions were chosen to measure the structure to obtain particular modes, which apparently could not be obtained when a single direction was conducted. Three accelerometers were attached to the structure and were roved symmetrically to provide symmetric mass distribution.

The bolt-jointed structure was measured using 16 channel LMS SCADAS acquisition system. The natural frequencies and mode shapes of the assembled structure were extracted using PolyMAX curve fitting method from the LMS software.

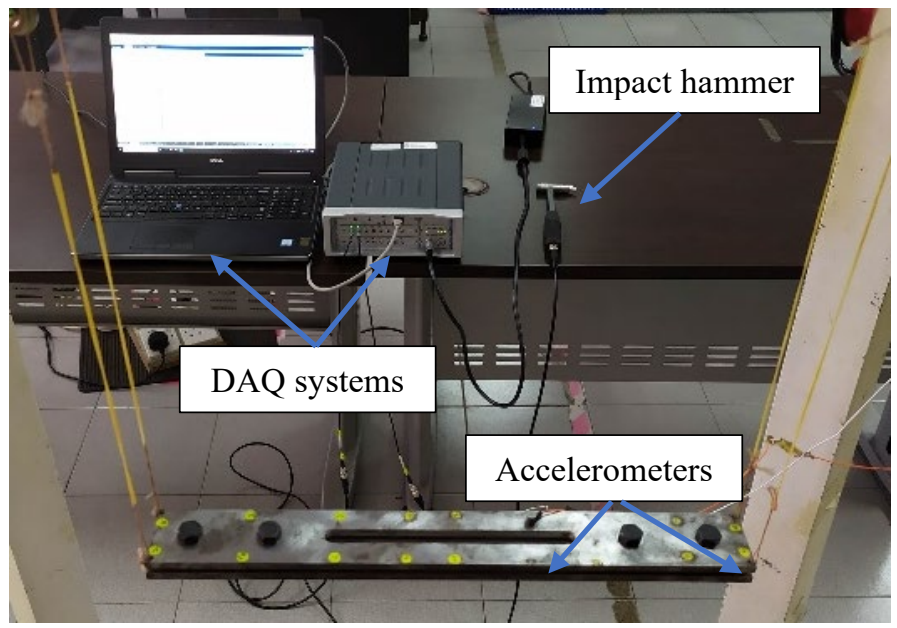

Figure 5. Experimental setup of assembled bolt-jointed structure.

\section{Stage 3: Development of Accurate Stochastic Model of Assembled Bolt-Jointed Structure}

\section{Stochastic modelling and updating of assembled bolt-jointed structure}

The stochastic finite element method (SFEM) is a mathematical tool to model structural uncertainties and randomness of a system such as on the bolt-jointed structure. The SFEM is an extension of the finite element method that incorporates random input parameters. The analytical model of SFEM can represent randomness in the structural parameters such as in geometry, material properties, joint interface and properties, experimental boundary conditions and many more. In order to study and analyse the structural inherent uncertainties and randomness, the SFEM use a statistical approach such as Monte Carlo Simulation (MCS) to characterise the response variability of the structure through the use of repeated samplings. The statistical parameters such as mean, variance, covariance and correlation coefficients of structural responses such as natural frequencies are used to assess the quality of the predicted stochastic model.

The stochastic model was developed by implementing the probability method and MCS with focusing on the influence of random input parameters, particularly on the joint interface of the bolt-jointed structure. In the probability theory, a domain of random parameter $\theta_{\mathrm{i}}$ is defined as any possible values and likelihood of the random parameter being inside a certain domain is given by a Probability Density Function (PDF), $f_{X}\left(\theta_{\mathrm{i}}\right)$. The PDF can be used to evaluate the probability of likelihood of a random parameter in a particular domain of interest. For instance, if bolt stiffness, $\theta_{\mathrm{i}}$ is a random parameter with $\operatorname{PDF} f_{X}\left(\theta_{\mathrm{i}}\right)$, the probability of the parameter is indicated as:

$$
P\left(a \leq \theta_{\mathrm{i}} \geq b\right)=\int_{a}^{b} f_{X}\left(\theta_{\mathrm{i}}\right) d x
$$


where $a$ is the lower bound and $b$ is the upper bound of random interval parameter and the probability that $\theta_{\mathrm{i}}$ falls in the interval can be written as $\left(a \leq \theta_{\mathrm{i}} \geq b\right)$. After defining a variation domain of the inputs, $n$ samples are generated from a PDF over the domain. For each random sample, a deterministically predicted response is defined and computed using a framework of finite element method as stated in Subsection STAGE 1 and STAGE 2. The random dynamic behaviour calculation of the SFEM principle in which bounded by PDF can be expressed as:

$$
\left(\overline{\mathbf{K}}-\bar{\omega}_{\mathrm{k}}^{2} \overline{\mathbf{M}}\right) \overline{\boldsymbol{\phi}}_{\mathbf{i}}=\mathbf{0}
$$

where $\overline{\mathbf{K}}$ and $\overline{\mathbf{M}}$ are the random stiffness and random mass matrices, meanwhile $\overline{\boldsymbol{\phi}}_{\mathbf{i}}$ is the $\mathrm{n} \times 1$ modal displacement vector (mode shapes), $\bar{\omega}_{\mathrm{k}}^{2}$ is the mean eigenvalue and $\bar{\omega}$ is the mean natural frequencies. Meanwhile, the covariance of natural frequencies can be obtained by

$$
\operatorname{Cov}\left(\omega_{\mathrm{k}}, \omega_{\mathrm{l}}\right)=\sum_{\mathrm{i}} \sum_{\mathrm{j}} \frac{\partial \bar{\omega}_{\mathrm{k}}}{\partial \theta_{\mathrm{i}}} \frac{\partial \bar{\omega}_{\mathrm{l}}}{\partial \theta_{\mathrm{j}}} \operatorname{Cov}\left(\theta_{\mathrm{i}}, \theta_{\mathrm{j}}\right)
$$

where the overbar parameters denote the mean values.

In order to optimise the stochastic model in light of experiment data, the stochastic model updating method using perturbation procedure is carried out. According to the deterministic based method, predicted parameters can be updated by solving Eq. (4). However, to take into account the variability in measurements of bolt-jointed structure in the model updating procedure, the modal parameters are represented as:

$$
\begin{gathered}
\mathrm{Z}_{\mathrm{m}}=\overline{\mathrm{Z}}_{\mathrm{m}}+\Delta \mathrm{Z}_{\mathrm{m}} \\
\mathrm{Z}_{\mathrm{j}}=\overline{\mathrm{Z}}_{\mathrm{j}}+\Delta \mathrm{Z}_{\mathrm{j}} \\
\theta_{\mathrm{j}}=\bar{\theta}_{\mathrm{j}}+\Delta \theta_{\mathrm{j}}
\end{gathered}
$$

where the overbar denotes as mean values and $\Delta \mathrm{Z}_{\mathrm{m}}$ and $\Delta \mathrm{Z}_{\mathrm{j}}$ are vectors of random variables. Meanwhile $\Delta \theta_{\mathrm{j}}$ is the variability in physical parameters at the $\mathrm{j}$-th iteration. The stochastic model updating formulation can be rewritten by substituting Eq.(11), Eq. (12), and Eq. (13) into Eq. (4) as follow:

$$
\hat{\theta}_{j+1}+\Delta \theta_{j+1}=\widehat{\theta}_{j}+\Delta \theta_{j}+\left(\widehat{T}_{j}+\Delta \mathbf{T}_{j}\right)\left(\hat{Z}_{m}+\Delta Z_{m}-\widehat{Z}_{j}-\Delta Z_{j}\right)
$$

and the transformation matrix $\mathbf{T}$ may be expressed as:

$$
\widehat{\mathbf{T}}_{\mathbf{j}}=\left(\widehat{\mathbf{S}}_{\mathbf{j}}^{\mathbf{T}} \mathbf{W}_{\varepsilon \varepsilon} \widehat{\mathbf{S}}_{\mathbf{j}}+\mathbf{W}_{\theta \theta}\right)^{-1} \hat{\mathbf{S}}_{\mathbf{j}}^{\mathbf{T}} \mathbf{W}_{\varepsilon \varepsilon}
$$

where $\hat{\mathbf{S}}_{\mathbf{j}}$ is the sensitivity matrix evaluated at mean structure parameters, meanwhile $\mathbf{W}_{\boldsymbol{\theta} \boldsymbol{\theta}}$ and $\mathbf{W}_{\boldsymbol{\varepsilon} \boldsymbol{\varepsilon}}$ are weighting matrices for parameters and experiment data.

In this work, the stochastic predicted model was developed using MCS type method by implemented Latin Hypercube Sampling (LSH) technique. The stochastic modelling technique was applied to the FE-Type 4 model in which the details of the FE model selections described in Section 5. Meanwhile, experimental data for stochastic model updating of the bolt-jointed structure was collected by disassembled and reassembled the bolt joints for 20 times with torque was set to $40 \mathrm{Nm}$ to provide variability in the experimental dynamic behaviour of the structure. For the sake of reducing structural variability, setup for all experimental series such as sensor locations, boundary conditions, excitation location and bolted joints torque remained in the same configuration.

\section{RESULTS AND DISCUSSION}

Development of an accurate and efficient stochastic dynamic behaviour prediction and parameters uncertainty propagation of bolt-jointed structure with concern to the model source of uncertainty from the structural stiffness properties was the aim of this study. There are three stages that have been introduced in the research works in which to describe and achieve the aim of this study. In the first stage, the analytical model of substructures elliptical plate A and elliptical plate B were modelled using the FE method. Then, the predicted dynamic behaviour such as natural frequencies and mode shapes of the substructures were obtained. Meanwhile, the experimental natural frequencies and mode shapes were obtained using experimental modal analysis (EMA). The results from the experimental work were used as a benchmark to evaluate the accuracy of the FE models. The results of the first ten natural frequencies obtained from the EMA and FEM of the substructures elliptical plate A and elliptical plate B were tabulated in Table 4 and Table 5. In Table 4, the comparison of natural frequencies between EMA and FEM of elliptical plate A recorded a total error of 35.33\%, meanwhile, for Table 5, the total error recorded for elliptical plate B was $32.21 \%$. The predicted mode shapes of elliptical plate A and elliptical plate B show a good correlation with experimental modes with the Modal Assurance Criterion 
(MAC) values of all modes were more than 0.9. However, the total errors of the substructures need to be minimised by using the model updating method.

Table 4. Comparison between experimental, FE and updated model of elliptical plate A.

\begin{tabular}{lcccccc}
\hline Mode & I & II & III & IV & V & VI \\
\cline { 2 - 7 } & $\begin{array}{c}\text { Experimental } \\
\text { frequencies } \\
(\mathrm{Hz})\end{array}$ & $\begin{array}{c}\text { FE frequencies } \\
(\mathrm{Hz})\end{array}$ & $\begin{array}{c}\text { Error (I-II/I) } \\
(\%)\end{array}$ & $\begin{array}{c}\text { Updated } \\
\text { frequencies }(\mathrm{Hz})\end{array}$ & $\begin{array}{c}\text { Error (I-IV/I) } \\
(\%)\end{array}$ & MAC \\
\hline 1 & 211.06 & 203.30 & 3.68 & 210.82 & 0.11 & 0.91 \\
2 & 604.93 & 580.67 & 4.01 & 602.10 & 0.47 & 0.95 \\
3 & 919.11 & 881.12 & 4.13 & 918.97 & 0.02 & 0.90 \\
4 & 1088.89 & 1048.80 & 3.68 & 1089.74 & 0.08 & 0.99 \\
5 & 1207.47 & 1156.50 & 4.22 & 1199.14 & 0.69 & 0.90 \\
6 & 1587.78 & 1540.50 & 2.98 & 1597.70 & 0.62 & 0.91 \\
7 & 1966.39 & 1921.80 & 2.27 & 1985.27 & 0.96 & 0.96 \\
8 & 2000.26 & 1914.90 & 4.27 & 1993.14 & 0.36 & 0.90 \\
Total error & & & 35.33 & & 3.31 & \\
\hline
\end{tabular}

The steepest gradient algorithm was used to optimise the influential parameters of the substructures. As shown in Figure 6, natural frequencies of both substructures were sensitive to Young's modulus, density and shear modulus of the substructures. However, density was not included in the updating process. This is because density is a measurable parameter, and thus, the true value of density can be calculated manually. The updated parameters data were tabulated in Table 6. By using the updating method, the total errors for predicted models of elliptical plate A and plate B managed to be minimised to $4.97 \%$ and $4.26 \%$, respectively.

Furthermore, result in Table 4 and Table 5 shows that, even though both substructures are two nominally identical structures, manufactured in the same production system, have similarity in term of geometrical and material properties; however, dynamic structural analysis is important to identify the precise dynamic behaviour of the substructures. The dynamic behaviour of both substructures is expected to be similar, however, due to the stochastic nature that exists among the substructures, the responses, particularly natural frequencies of the substructures, is slightly different to each other $[21]-[24]$.

Table 5. Comparison between experimental, FE and updated model of elliptical plate B.

\begin{tabular}{lcccccc}
\hline \multirow{2}{*}{ Mode } & I & II & III & IV & V & VI \\
\cline { 2 - 7 } & $\begin{array}{c}\text { Experimental } \\
\text { frequencies (Hz) }\end{array}$ & $\begin{array}{c}\text { FE frequencies } \\
(\mathrm{Hz})\end{array}$ & $\begin{array}{c}\text { Error (I-II/I) } \\
(\%)\end{array}$ & $\begin{array}{c}\text { Updated } \\
\text { frequencies (Hz) }\end{array}$ & $\begin{array}{c}\text { Error (I-IV/I) } \\
(\%)\end{array}$ & MAC \\
\hline 1 & 212.00 & 203.72 & 3.90 & 210.94 & 0.50 & 0.93 \\
2 & 604.27 & 581.91 & 3.70 & 602.57 & 0.28 & 0.92 \\
3 & 911.44 & 882.86 & 3.14 & 911.28 & 0.02 & 0.98 \\
4 & 1086.10 & 1051.13 & 3.22 & 1087.20 & 0.10 & 0.90 \\
5 & 1203.50 & 1159.04 & 3.69 & 1200.16 & 0.28 & 0.97 \\
6 & 1592.66 & 1543.64 & 3.08 & 1598.23 & 0.35 & 0.97 \\
7 & 1972.10 & 1925.75 & 2.35 & 1987.21 & 0.77 & 0.91 \\
8 & 1996.49 & 1918.90 & 3.89 & 1993.87 & 0.13 & 0.93 \\
& Total Error & & 32.21 & & 2.42 & \\
\hline
\end{tabular}

Table 6. Updated parameters for elliptical plate A and plate B.

\begin{tabular}{lcccc}
\hline \multirow{2}{*}{ Parameters } & \multicolumn{2}{c}{ Elliptical plate A } & \multicolumn{2}{c}{ Elliptical plate B } \\
\cline { 2 - 5 } & Initial & Updated & Initial & Updated \\
\hline Young's modulus, E $\mathrm{B}_{\mathrm{B}}(\mathrm{GPa})$ & 200 & 215 & 200 & 214 \\
Shear modulos, $\mathrm{G}_{\mathrm{B}}(\mathrm{GPa})$ & 81 & 83.72 & 81 & 81.92 \\
\hline
\end{tabular}

After successfully developed an accurate predicted model for the substructures, the research works were continued to Stage 2. Stage 2 comprises modelling procedure for assembled bolt-jointed structure. In this stage, the updated FE models of elliptical plate A and plate B were joined together to form an assembled bolt-jointed structure as described in subsection Stage 2. There are four types of element connectors used to model the bolt joint interface. Each of the models is identified as RBE bolt-jointed FE model (FE Type 1), CBAR bolt-jointed FE model (FE Type 2), CELAS bolt-jointed FE model (FE Type 3) and CBUSH bolt-jointed FE model (FE Type 4), respectively. The aim in this stage is to identify the most appropriate FE models to represent the assembled bolt-jointed structure for stochastic modelling and updating procedure. This is because not all element connectors available in commercial FE software are suitable to represent as bolt joint in the stochastic analysis due to the limited sub-parameters to be used in stochastic modelling and updating [25]. The comparison of natural frequencies between EMA and the four initial FE model were tabulated in Table 7. It was found that the total errors recorded for FE-Type 1, FE-Type 2, FE-Type 3 and FE-Type 4 were 30.70 \%, $26.33 \%, 26.28 \%$ and $25.28 \%$, respectively. From the results, it can be seen that the errors between EMA and all four FE models were not 
having much different between each other. Nevertheless, the total errors for initial predictions of the bolt-jointed structure did not have significant effects in this stage. This is because the selection of the most appropriate model to represent the bolt-jointed structure for the stochastic modelling depends on the feasibility of the element connectors to represent the uncertain nature of the assembled structure.

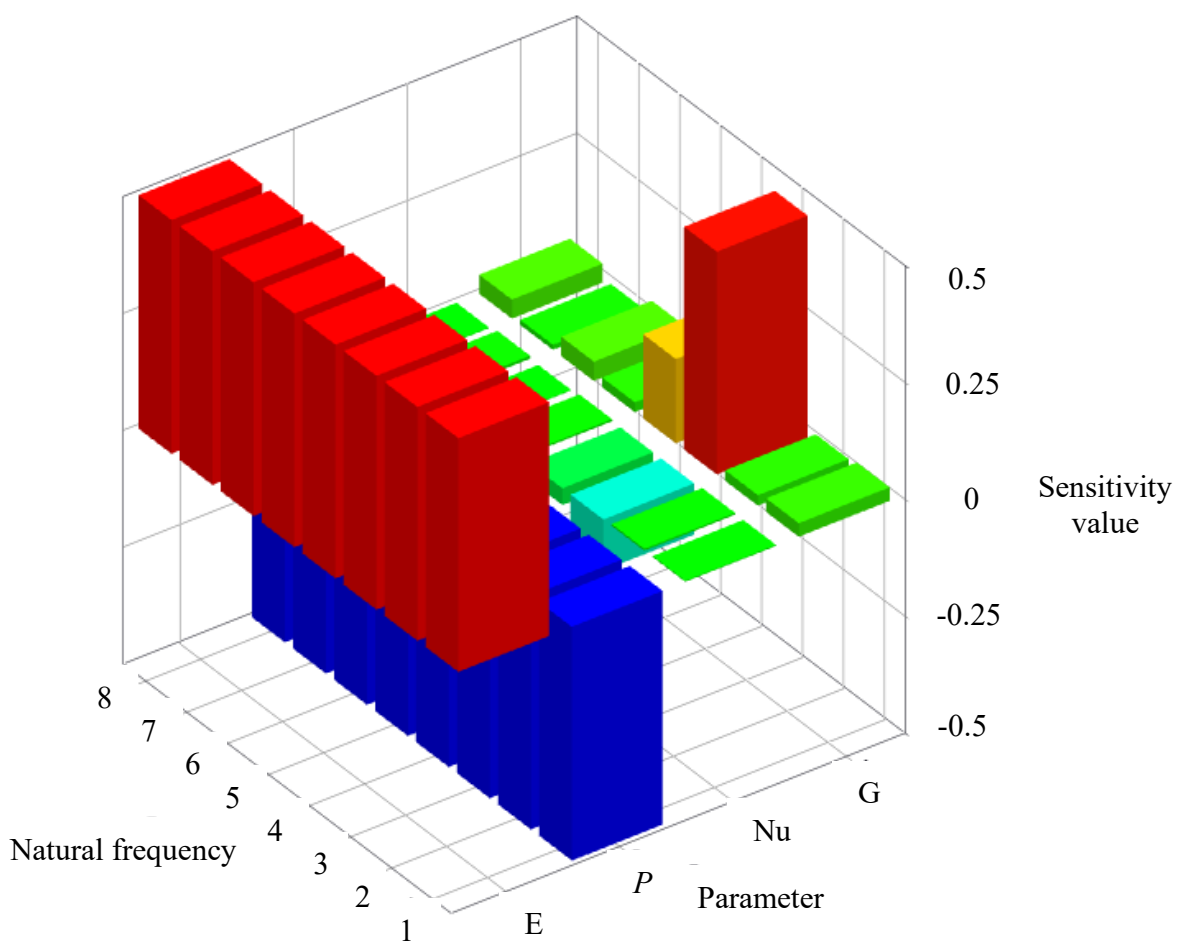

Figure 6. Sensitivity chart of substructures.

Even though the total errors were not much different, however, the FE-Type 4 was the most appropriate model to be used to represent bolt-jointed structure. Table 8 shows the mode shapes of the FE-Type 4 model. This is because CBUSH element connector is the most detailed spring element in which the joint stiffness can be provided in 6 degree of freedom (DoF) [26]. By doing so, the uncertainty study for the joint stiffness can be expanded into different DoF. The advantage of a detailed spring element could not be acquired if element connectors such as RBE, CBAR or CELAS were used in the stochastic modelling and updating of bolt-jointed structure. Meanwhile, the spring element such as CELAS was not suitable to represent bolt joint because it only provided one translation DoF stiffness in which were not sufficient to study the stochastic nature of the structure. Moreover, the FE-Type 1 (RBE connector) was not suitable to represent a bolt joint due to its limitation in providing optimum stiffness. Theoretically, the RBE connector is develop based on 1D rigid and containing infinite stiffness properties in all directions [27]-[30]. Specifically, the stiffness properties of the RBE connector cannot be controlled nor updated.

Table 7. Comparison of experimental and FE models of assembled bolt-jointed structure.

\begin{tabular}{|c|c|c|c|c|c|c|c|c|c|}
\hline & I & II & III & IV & $\mathrm{V}$ & VI & VII & VIII & VIIII \\
\hline Mode & $\begin{array}{c}\text { EMA } \\
(\mathrm{Hz})\end{array}$ & $\begin{array}{c}\text { Type } 1 \\
(\mathrm{RBE}) \\
(\mathrm{Hz})\end{array}$ & $\begin{array}{c}\text { Error } \\
(\mathrm{I}-\mathrm{II} / \mathrm{I}) \\
(\%)\end{array}$ & $\begin{array}{c}\text { Type } 2 \\
(\mathrm{CBEAM}) \\
(\mathrm{Hz})\end{array}$ & $\begin{array}{c}\text { Error } \\
(\mathrm{I}-\mathrm{VI} / \mathrm{I}) \\
(\%)\end{array}$ & $\begin{array}{c}\text { Type } 3 \\
\text { (CELAS) } \\
(\mathrm{Hz})\end{array}$ & $\begin{array}{c}\text { Error } \\
(\mathrm{I}-\mathrm{VI} / \mathrm{I}) \\
(\%)\end{array}$ & $\begin{array}{c}\text { Type } 4 \\
(\mathrm{CBUSH}) \\
(\mathrm{Hz})\end{array}$ & $\begin{array}{c}\text { Error } \\
(\mathrm{I}-\mathrm{VIII} / \mathrm{I}) \\
(\%)\end{array}$ \\
\hline 1 & 496.76 & 530.29 & 6.75 & 524.18 & 5.52 & 526.45 & 5.98 & 526.24 & 5.94 \\
\hline 2 & 619.44 & 647.85 & 4.58 & 634.13 & 2.37 & 635.35 & 2.57 & 637.84 & 2.97 \\
\hline 3 & 661.25 & 659.24 & 0.30 & 667.66 & 0.97 & 666.95 & 0.86 & 659.11 & 0.32 \\
\hline 4 & 860.27 & 892.90 & 3.79 & 908.24 & 5.58 & 904.97 & 5.20 & 891.97 & 3.68 \\
\hline 5 & 904.77 & 909.22 & 0.49 & 911.18 & 0.71 & 909.48 & 0.52 & 906.18 & 0.16 \\
\hline 6 & 1063.86 & 1075.97 & 1.14 & 1071.90 & 0.76 & 1071.90 & 0.76 & 1072.84 & 0.84 \\
\hline 7 & 1462.71 & 1542.74 & 5.47 & 1529.00 & 4.53 & 1529.09 & 4.54 & 1539.64 & 5.26 \\
\hline 8 & 1552.29 & 1629.44 & 4.97 & 1591.60 & 2.53 & 1590.60 & 2.47 & 1599.42 & 3.04 \\
\hline 9 & 1747.00 & 1742.22 & 0.27 & 1765.30 & 1.05 & 1765.90 & 1.08 & 1741.76 & 0.30 \\
\hline 10 & 1939.81 & 1996.62 & 2.93 & 1984.70 & 2.31 & 1984.70 & 2.31 & 1993.60 & 2.77 \\
\hline & Total error & & 30.70 & & 26.33 & & 26.28 & & 25.28 \\
\hline
\end{tabular}


Table 8. Comparison of mode shape between experimental and FE-Type 4 model.

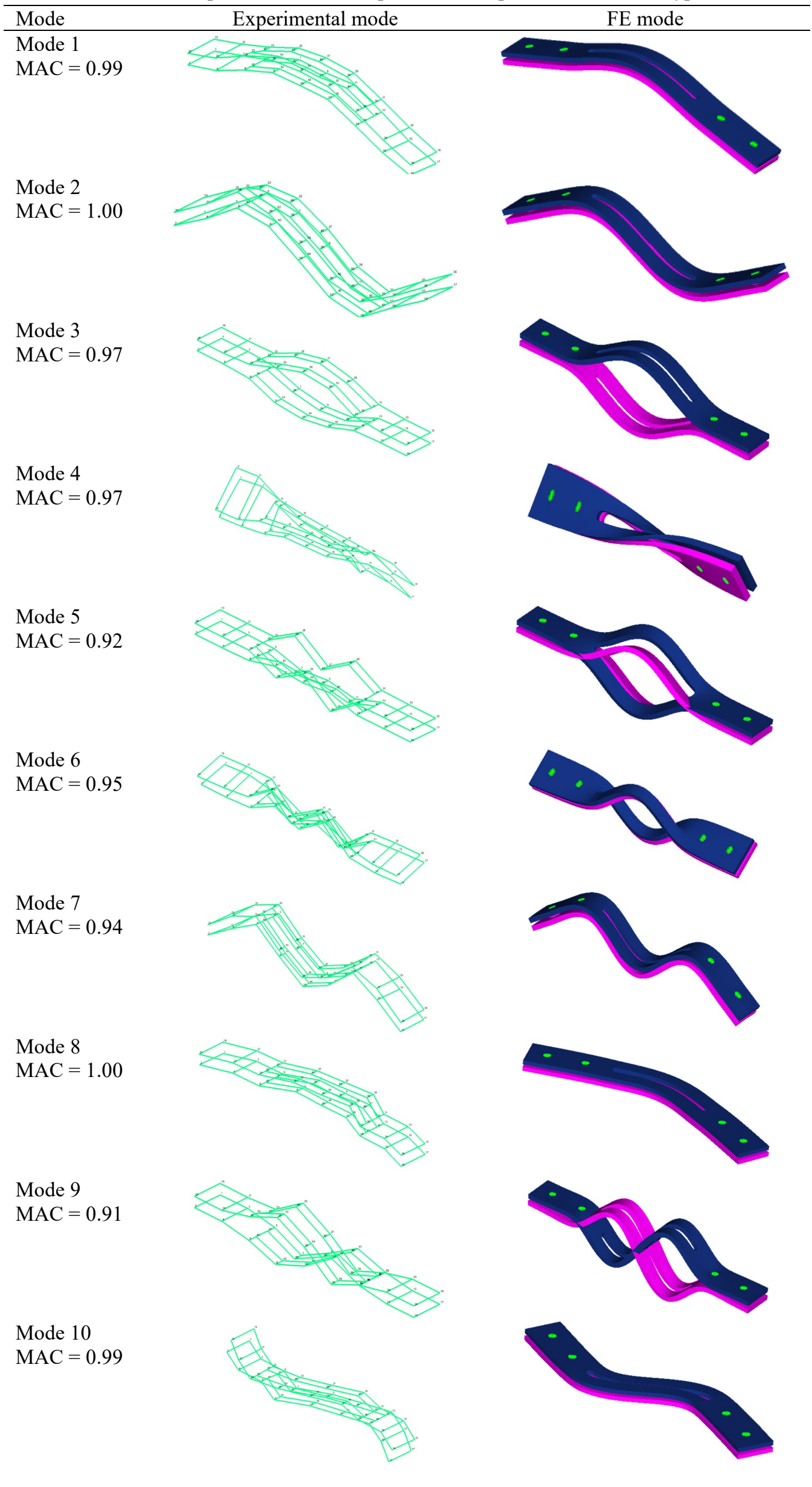


In Stage 3, the FE-Type 4 model was used as the initial prediction model to study the stochastic nature due to the bolt joint. The stochastic model was developed by using the Latin Hypercube Sampling technique by randomised the bolt joints stiffness using 50 samplings data. Meanwhile, the variability in the experimental data was provided by reassembled and dissembled the bolt joints 20 times to produce randomness joint stiffness. Variability of the natural frequencies for the predicted model and experimental data were illustrated in the statistical characteristic such as mean and standard deviation. The standard deviation (Std) was used to measures the dispersion of a dataset relative to its mean [31]. The experimental, initial predicted and updated means and standard deviations of the assembled bolt-jointed structure were given in Table 9. Ten natural frequencies were analysed in this study. It can be seen that the comparison of the results between experimental and initial stochastic estimations was not in good agreement. The total errors recorded for means frequencies was $22.33 \%$.

Table 9. Comparison of stochastic data of assembled bolt-jointed structure.

\begin{tabular}{|c|c|c|c|c|c|c|c|c|c|c|}
\hline \multirow{3}{*}{ Mode } & I & II & III & IV & $\mathrm{V}$ & VI & VII & VIII & VIIII & XI \\
\hline & \multicolumn{2}{|c|}{$\begin{array}{c}\text { Experimental } \\
\text { frequencies }(\mathrm{Hz})\end{array}$} & \multicolumn{2}{|c|}{$\begin{array}{c}\text { SFE-Type } 4 \\
\text { frequencies }(\mathrm{Hz})\end{array}$} & \multicolumn{2}{|c|}{ Error (\%) } & \multicolumn{2}{|c|}{$\begin{array}{c}\text { Updated } \\
\text { frequencies }(\mathrm{Hz})\end{array}$} & \multicolumn{2}{|c|}{ Error $(\%)$} \\
\hline & Mean & Std & Mean & Std & Mean & Std & Mean & Std & Mean & Std \\
\hline 1 & 508.28 & 6.49 & 505.39 & 7.95 & 0.57 & 22.50 & 506.39 & 6.91 & 0.37 & 6.47 \\
\hline 2 & 627.30 & 4.47 & 611.67 & 9.63 & 2.49 & 115.44 & 619.67 & 5.94 & 1.22 & 32.89 \\
\hline 3 & 669.57 & 5.80 & 644.17 & 10.05 & 3.79 & 73.28 & 654.85 & 7.41 & 2.20 & 27.76 \\
\hline 4 & 867.96 & 4.84 & 857.83 & 13.51 & 1.17 & 179.13 & 859.81 & 5.61 & 0.94 & 15.91 \\
\hline 5 & 911.87 & 4.39 & 873.76 & 13.96 & 4.18 & 218.00 & 892.1 & 6.22 & 2.17 & 41.69 \\
\hline 6 & 1073.15 & 6.28 & 1036.15 & 16.62 & 3.45 & 164.65 & 1059.14 & 9.32 & 1.31 & 48.41 \\
\hline 7 & 1470.24 & 4.26 & 1475.20 & 23.22 & 0.34 & 445.07 & 1475.9 & 9.25 & 0.38 & 117.14 \\
\hline 8 & 1558.34 & 3.84 & 1530.30 & 24.12 & 1.80 & 528.13 & 1542.51 & 8.99 & 1.02 & 134.11 \\
\hline 9 & 1753.20 & 3.63 & 1703.56 & 26.60 & 2.83 & 632.78 & 1731.11 & 7.36 & 1.26 & 102.75 \\
\hline 10 & 1947.15 & 3.78 & 1913.86 & 30.17 & 1.71 & 698.15 & 1922.65 & 9.42 & 1.26 & 149.21 \\
\hline \multicolumn{3}{|c|}{ Total error } & & & 22.33 & & & & 12.13 & \\
\hline
\end{tabular}

Meanwhile, the initial standard deviation prediction shows that very high disagreement between sets of initial sampling data. This situation occurs due to two problems: (1) inaccurate initial FE model and (2) random sampling problem. For the first problem, the initial assembled FE model (FE-Type 4) was not undergo updating process (only substructures were updated); therefore, the bolt stiffness and the contact stiffness were only based on estimation and calculation using swift formulation [32]. The FE model still at low level of accuracy. Meanwhile, for the second problem, the LHS random sampling basically depends on the range of randomised parameters. The range of randomised parameters must be precise to provide accurate sampling data. However, the disagreement in the stochastic data can be minimised by using the stochastic model updating method. Table 9 shows the comparison between experimental mean frequencies and updated mean frequencies. It can be seen that the total error of the initial prediction has been dropped considerably from $22.33 \%$ to $12.13 \%$. The standard deviation for the stochastic updated frequencies shows considerable improvement. These results highlight the capabilities of stochastic model updating in predicting and improvising predicted frequencies with the influence of uncertainties or randomness joint stiffness.

\section{CONCLUSION}

Procedures to develop an accurate stochastic dynamic behaviour prediction and parameters uncertainty propagation of bolt-jointed structure with concern to model source of uncertainty contributed from stiffness properties of bolt joints interface and contact zones using stochastic model updating method has been presented. Even though there are significant studies that can be referred regarding RBE, CBAR, CELAS and CBUSH representation as bolt joint. However, it can be summarised that the selection of the element connectors is depended on the type of analysis and applications. For the case stochastic modelling and updating, CBUSH is much more appropriate to represent as bolt joints. Nevertheless, the stochastic predictions were not in good agreement with the sets of experimental data. This is due to the inaccuracy of initial FE prediction and random sampling problems. Therefore, stochastic model updating need to be performed to improve the correlation of FE results with experimental data.

\section{ACKNOWLEDGEMENT}

The authors wish to acknowledge the Malaysia Ministry of Higher Education (MOHE), Research Management Institute (RMI) and Institute of Graduate Studies of Universiti Teknologi MARA (UiTM) for providing financial support for this research through the fundamental research grant scheme (FRGS) 600-IRMI/FRGS 5/3 (172/2019). They would also like to extend their sincere gratitude for the support and help given by all SDAV members.

\section{REFERENCES}

[1] Jalali H, Khodaparast HH, Friswell MI, et al. Finite element modelling and updating for bolted lap joints. AIP Conference Proceedings 2018;141(1):202-222. 
[2] Omar R, Rani MNA, Yunus MA, et al. Investigation of Mesh Size effect on dynamic behaviour of an assembled structure with bolted joints using finite element method. International Journal of Automotive and Mechanical Engineering 2018;15(3):56955709.

[3] Aziz Shah MAS, Yunus MA, Abdul Rani MN. The application of design of experiment method in finite element model updating for estimating uncertainties of Laser Stitch Welded Structure. IOP Conference Series: Materials Science and Engineering 2020;788(1).

[4] Kim J, Yoon J-C, Kang B-S. Finite element analysis and modeling of structure with bolted joints. Applied Mathematical Modelling 2007;31(5):895-911.

[5] Guzas E, Behan K, Davis J. 3D finite element modeling of single bolt connections under static and dynamic tension loading. Shock and Vibration 2015;2015.

[6] Izham MHN, Abdullah NAZ, Zahari SN, Sani MSM. Structural dynamic investigation of frame structure with bolted joints. MATEC Web of Conferences 2016;90:1-9.

[7] Ming Z, Qintao G, Lin Y, Baoqiang Z. Finite element model updating of jointed structure based on modal and strain frequency response function. Journal of Mechanical Science and Technology 2019;33(10):4583-4593.

[8] Ahmadian H, Jalali H. Identification of bolted lap joints parameters in assembled structures. Mechanical Systems and Signal Processing 2007;21(2):1041-1050.

[9] Zhai X, Fei CW, Choy YS, Wang JJ. A stochastic model updating strategy-based improved response surface model and advanced Monte Carlo simulation. Mechanical Systems and Signal Processing 2017;82:323-338.

[10] Omar R, Rani MNA, Yunus MA. Finite Element Modelling and Updating of a Bolted Structure Using Elements Representing the Stiffness Members, Bolts and Affected Areas of the Joints. Journal of Physics: Conference Series 2019;1262(1).

[11] Adel F, Shokrollahi S, Jamal-Omidi M, Ahmadian H. A model updating method for hybrid composite/aluminum bolted joints using modal test data. Journal of Sound and Vibration 2017;396:172-185.

[12] Baramsky N, Seibel A, Schlattmann J. Friction-induced vibrations during tightening of bolted joints—Analytical and experimental results. Vibration 2018;1(2):312-337.

[13] Arregui-Mena JD, Margetts L, Mummery PM. Practical application of the stochastic finite element method. Archives of Computational Methods in Engineering 2016;23(1):171-190.

[14] Patelli E, Govers Y, Broggi M, et al. Sensitivity or Bayesian model updating: a comparison of techniques using the DLR AIRMOD test data. Archive of Applied Mechanics 2017;87(5):905-925.

[15] Abu Husain N, Haddad Khodaparast H, Ouyang H. Parameter selection and stochastic model updating using perturbation methods with parameter weighting matrix assignment. Mechanical Systems and Signal Processing 2012;32:135-152.

[16] Shah MASA, Yunus MA, Rani MNA, et al. Structural modification and evaluation using finite element reconciliation method. AIP Conference Proceedings 2019;2059(1): 020007.

[17] Aziz Shah MAS, Yunus MA, Abdul Rani MN, Saman AM, M. Sani MS, Mohd Zin MS. The effect of laser stitch welding residual stress on the dynamic behaviour of thin steel structure. Journal of Mechanical Engineering and Sciences 2019;13(4):5780-5790.

[18] Bahari AR, Yunus MA, Abdul Rani MN, et al. Investigation on the effects of suspension stiffness using experimental modal analysis and finite element model updating. IOP Conference Series: Materials Science and Engineering 2019;506(1): 012043.

[19] Bartilson DT. Model updating in structural dynamics: advanced parametrisation, optimal regularisation, and symmetry considerations. PhD Thesis, Columbia University, USA, 2019.

[20] Omar R, Rani MNA, Yunus MA, et al. Efficient finite element modelling for the investigation of the dynamic behaviour of a structure with bolted joints. AIP Conference Proceedings 2018;1952:020082.

[21] Govers Y, Haddad Khodaparast H, Link M, Mottershead JE. A comparison of two stochastic model updating methods using the DLR AIRMOD test structure. Mechanical Systems and Signal Processing 2015;52-53(1):105-114.

[22] Rui Q, Ouyang H, Wang HY. An efficient statistically equivalent reduced method on stochastic model updating. Applied Mathematical Modellin 2013;37(8):6079-6096.

[23] Jalali H, Khodaparast HH, Madinei H, Friswell MI. Stochastic modelling and updating of a joint contact interface. Mechanical Systems and Signal Processing 2019;129:645-658.

[24] Scinocca F, Nabarrete A. Parametric stochastic analysis of a piezoelectric vibration absorber applied to automotive body structure. Journal of Vibration Engineering \& Technologies 2020;8:199-213.

[25] Zhan M, Guo Q, Yue L, Zhang B. Modeling and stochastic model updating of bolt-jointed structure. Shock and Vibration 2018;2018:8354249.

[26] Gibanica M, Abrahamsson TJS, Olsson M. Model updating of multiple nominally identical car components. Experimental Techniques 2020;44(4):391-407.

[27] Shah MASA, Yunus MA, Saman AM, et al. Finite element model updating of laser stitch welded structure. IOP Conference Series: Materials Science and Engineering 2019;506:012010.

[28] Yunus MA, Rani MNA, Sani MSM, Shah MASA. Finite element model updating of riveted joints of simplified model aircraft structure. AIP Conference Proceedings 2018;1952(1):20013.

[29] Zin MSM, Rani MNA, Yunus MA, et al. Modal based updating for the dynamic behaviour of a car trunk lid. AIP Conference Proceedings 2019;2059(1): 020001. 
[30] Yunus MA, Ouyang H, Rani MNA, Isa AAM. Finite element modelling and updating of bolted joints in a thin sheet metal structure. In: Crocker MJ, Pawelczyk M, Paosawatyanyong B, editor. 20th International Congress on Sound and Vibration 2013 (ICSV 2013), Volume 2. New York: International Institute of Acoustics \& Vibration, 2013, p 1146-1152.

[31] Fang SE, Zhang QH, Ren WX. Parameter variability estimation using stochastic response surface model updating. Mechanical Systems and Signal Processing 2014;49(1-2):249-263.

[32] Mara V, Haghani R, Al-Emrani M. Improving the performance of bolted joints in composite structures using metal inserts. Journal of Composite Materials 2016;50(21):3001-3018. 